CERN-ECP/96-05

30 May 1996

\title{
STUDY OF CHARGE COLLECTION AND NOISE IN NON-IRRADIATED AND IRRADIATED SILICON DETECTORS
}

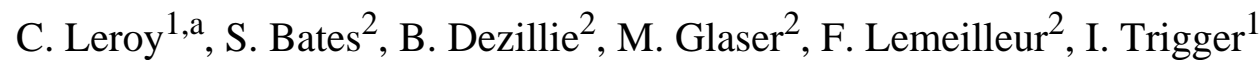

\begin{abstract}
The charge collection and noise were studied in non-irradiated and irradiated silicon detectors as a function of temperature (T), shaping time $(\theta)$ and fluence $(\Phi)$, up to about $1.2 \times 10^{14}$ protons per $\mathrm{cm}^{2}$, for minimum-ionizing electrons yielded by a ${ }^{106} \mathrm{Ru}$ source. The noise of irradiated detectors is found to be dominated for short shaping times $(\theta \leq 50 \mathrm{~ns})$ by a series noise component, while for longer shaping times $(\theta \geq 80 \mathrm{~ns})$ a parallel noise component (correlated with the reverse current) prevails. For non-irradiated detectors, where the reverse current is three orders of magnitude smaller compared with irradiated detectors, the series noise dominates over the whole range of shaping times investigated (20-150 ns). A signal degradation is observed for irradiated detectors. However, the signal can be distinguished from noise, even after a fluence of about $1.2 \times 10^{14}$ protons per $\mathrm{cm}^{2}$, at a temperature of $6{ }^{\circ} \mathrm{C}$ and with a shaping time typical of the LHC inter-bunch crossing time (20-30 ns). The measurement of the signal as a function of voltage shows that irradiated detectors depleted at $50 \%$ of the full depletion voltage can still provide a measurable signal-to-noise ratio.
\end{abstract}

Presented at the International Conference on Radiation Effects on Semiconductor Materials, Detectors and Devices, Florence, 6-8 March 1996.

1) University of Montreal, Montreal, Canada.

2) CERN, Geneva, Switzerland.

a) On Sabbatical leave at the University of Rome, La Sapienza, Rome, Italy. 


\section{INTRODUCTION}

Silicon detectors of various sizes will be used as active elements of tracking systems and calorimeters (preshower) to be operated in future LHC (Large Hadron Collider) experiments $[1,2]$. The silicon detectors will be exposed to high fluences of particles resulting from head-on collisions of $7 \mathrm{TeV}$ protons at an expected peak luminosity of $1.0 \times 10^{34} \mathrm{~cm}^{-2} \mathrm{~s}^{-1}$. The products of $\mathrm{p}-\mathrm{p}$ interactions, local beam losses, beam-gas interactions are then expected to inflict damage on silicon detectors. Irradiation creates damaged regions in the silicon bulk acting as electrically active defects with deep levels in the forbidden gap. The generation of these additional traps decreases the carrier lifetime and increases the reverse current. For fluences of the order of $10^{14}$ particles $/ \mathrm{cm}^{2}$, i.e. fluences corresponding to 10 years of LHC operation, the reverse current can reach values in the range of several tens of microamperes per $\mathrm{cm}^{2}$. However, the strong temperature dependence of the reverse current allows it to be minimized by operating the detectors at low temperature. The full depletion voltage is the minimal voltage to be applied in order to fully deplete the detector. Changes in the doping concentration (donors and/or acceptors) affect the value of the full depletion voltage which can reach very high (and unpractical) values after several years of the detector operation in high radiation environment. It is possible that after several years of operation at LHC, part of the detectors will have to be operated without being fully depleted. The LHC mode of operation itself brings another constraint since $\mathrm{p}-\mathrm{p}$ collisions will take place every $25 \mathrm{~ns}$. This short inter-bunch crossing time has the consequence that the silicon detectors have to be operated with shaping times as short as $25 \mathrm{~ns}$, at least at the first trigger level.

This article presents the results of a study of the charge collection and noise in nonirradiated and irradiated silicon detectors taking into account the various constraints imposed by their operation at the LHC, as described above. The response of silicon detectors and noise induced by minimum-ionizing electrons $(\geq 2 \mathrm{MeV})$ from a ${ }^{106} \mathrm{Ru}$ source were measured. A detailed investigation of the various noise components, performed as a function of fluence, temperature, and shaping time, allows one to understand the signal-to-noise ratio behaviour.

\section{EXPERIMENTAL SET-UP}

Non-irradiated and irradiated high-resistivity detectors were used for the study. They have been processed by Micron Semiconductor Ltd ( $1 \mathrm{~cm}^{2}$ area, square-shaped) and Canberra Ltd $\left(0.82 \mathrm{~cm}^{2}\right.$ area, disk-shaped) from $\mathrm{n}$-type silicon material $\mathrm{p}^{+}$implanted on one side to form the junction and $\mathrm{n}^{+}$on the other side to form the ohmic contact. The detectors have a thickness ranging from 143 to $996 \mu \mathrm{m}$ and a resistivity extending from 1.6 to $35.6 \mathrm{k} \Omega \mathrm{cm}$. The capacitance $\mathrm{C}$ of these detectors in the parallel plate capacitor approximation is given by

$$
\mathrm{C}=\varepsilon \mathrm{A} / \mathrm{w}
$$

where $\mathrm{A}$ and $\mathrm{w}$ are the detector area and thickness, respectively; $\varepsilon=1.05 \mathrm{pf} / \mathrm{cm}$ is the silicon dielectric constant. The depletion voltage $V_{d}$ as a function of the depletion depth $X_{d}$ is given by

$$
\mathrm{V}_{\mathrm{d}}=\mathrm{X}_{\mathrm{d}}^{2} / 2 \varepsilon \mu \rho=\mathrm{q}\left|\mathrm{N}_{\mathrm{eff}}\right| \mathrm{X}_{\mathrm{d}}^{2} / 2 \varepsilon
$$

where $\rho$ is the bulk resistivity, $\mu$ is the carrier mobility $\left(\mu_{\mathrm{e}}=1500 \mathrm{~cm}^{2} / \mathrm{V}\right.$.s and $\mu_{\mathrm{h}}=$ $450 \mathrm{~cm}^{2} / \mathrm{V} . \mathrm{s}$; [3]), q is the electric charge. $\mathrm{N}_{\text {eff }}$ is the effective dopant concentration $\left(\mathrm{N}_{\mathrm{eff}}=\mid \mathrm{N}_{\text {do- }}\right.$ nors $\left.-\mathrm{N}_{\text {acceptors }}\right)$. The energy deposited by a minimum-ionizing particle (m.i.p) in a silicon de- 
tector is about $80 \mathrm{keV}$ per $300 \mu \mathrm{m}$ of silicon corresponding to about 22000 electron-hole pairs created per $300 \mu \mathrm{m}$ of silicon.

Detectors were irradiated by $24 \mathrm{GeV} / c$ protons at various fluences (up to $1.2 \times$ $10^{14} \mathrm{p} \mathrm{cm}^{-2}$ ) at room temperature in the T7 beam at the CERN PS.

The signal response induced by minimum-ionizing electrons from a ${ }^{106} \mathrm{Ru}$ source and noise were studied for non-irradiated and irradiated detectors. The silicon detector under study was located inside a test box with the ${ }^{106} \mathrm{Ru}$ source fitted in its cover. The source was above the geometrical centre of the detector and collimated to a $5 \mathrm{~mm}$ diameter electron beam. The temperature inside the test box was controlled by a water cooling system allowing the selection of temperatures from 6 to $25{ }^{\circ} \mathrm{C}$. Nitrogen was flowing through the box to prevent water condensation on the detectors at low temperatures. The current pulse induced by electrons in the detector was detected with a Canberra 2003BT charge pre-amplifier used in the energy output mode. Sets of data were taken with a shaping time of $100 \mathrm{~ns}$. Then, the amplification and shaping were ensured by an Ortec 450 research amplifier. The shaping integration and differentiation were $100 \mathrm{~ns}$, with a gain of 500. The polarity of the input signals was negative. The output signals were in the $-3 \mathrm{~V}$ range. The pulse energy was measured with a peak sensing Lecroy ADC 2259A for a $500 \mathrm{~ns}$ gate triggered by the coincidence signal of two photomultipliers detecting photons produced in a scintillator of $1 \mathrm{~cm}^{2}$ area placed behind an iron absorber ( $\sim 0.5 \mathrm{~mm}$ thick) allowing the selection of minimum-ionizing electrons with an energy $\geq 2 \mathrm{MeV}$. For the measurements as a function of the shaping time, the Ortec 450 research amplifier was replaced by a CR-RC2 module, and a Lecroy 9360 fast digital oscilloscope equipped with histogramming facilities ensured the peak energy data acquisition.

\section{CHARGE COLLECTION STUDY}

The signal $\mathrm{S}$ of a silicon detector traversed by minimum-ionizing electrons is the most probable energy deposition, after pedestal subtraction. The pedestal distributions were obtained from special data-taking runs where the detector signal was delayed outside the trigger gate. The noise $\mathrm{N}$ is measured as the r.m.s. of the pedestal distribution fitted to a Gaussian distribution.

The standard thickness of silicon detectors envisaged for the LHC is $300 \mu \mathrm{m}$ [1]. For nonirradiated detectors, it is possible to enumerate some facts showing this choice as a good compromise. Thinner detectors present less volume and therefore smaller reverse current. The value of their full depletion voltage $\mathrm{V}_{\mathrm{fd}}$ is also smaller [see Eq. (2)]. As an example, for $200 \mu \mathrm{m}$ $(300 \mu \mathrm{m})$ thick detectors, $\mathrm{V}_{\mathrm{fd}} \sim 12 \mathrm{~V}(27 \mathrm{~V})$ and $84 \mathrm{~V}(190 \mathrm{~V})$ for $\rho=10 \mathrm{k} \Omega \mathrm{cm}$ and $1.5 \mathrm{k} \Omega \mathrm{cm}$, respectively. However, the signal-to-noise ratio $\mathrm{S} / \mathrm{N}$ for thin detectors is smaller than for thicker detectors. S/N measured as a function of the detector thickness is shown in Fig. 1. S/N is seen to increase linearly with the detector thickness. For instance, $\mathrm{S} / \mathrm{N} \sim 13$ and 20 for $200 \mu \mathrm{m}$ and $300 \mu \mathrm{m}$ thick detectors, respectively. The noise for non-irradiated detectors has been measured as a function of the detector thickness. The results are reported in Fig. 2 and show that the noise decreases with increasing detector thickness. The noise of non-irradiated detectors can be expressed as the sum of several components:

$$
\mathrm{N}=\left(\mathrm{N}_{\text {ir }}^{2}+\mathrm{N}_{\text {cap }}^{2}+\mathrm{N}_{\mathrm{d}}^{2}\right)^{1 / 2}
$$

where $\mathrm{N}_{\text {cap }}$ is the noise induced by the detector capacitance and contains also the preamplifier noise. $\mathrm{N}_{\mathrm{ir}}$ is the noise induced by the reverse current. $\mathrm{N}_{\mathrm{d}}$ is the detector intrinsic noise and also 
contains possible unknown sources of noise. The noise of non-irradiated detectors is dominated by the capacitance noise $\mathrm{N}_{\text {cap }}$ as shown in Fig. 2. The noise dependence as a function of capacitance was measured by replacing the silicon detectors in the test box by capacitances of known value. $\mathrm{N}_{\text {cap }}$ is found to decrease with an increasing thickness of the detector [Eq. (1)].

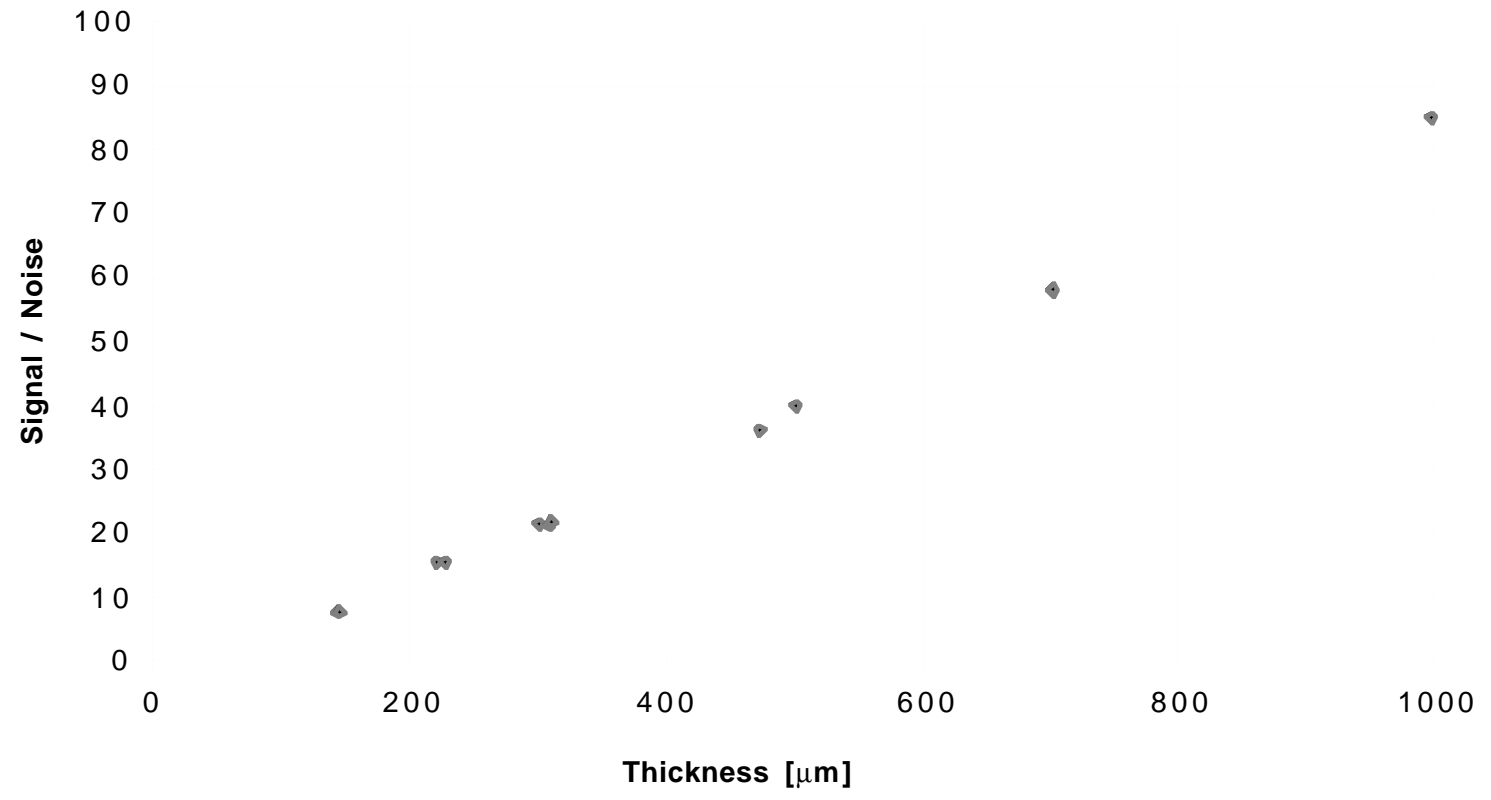

Fig. 1 The signal-to-noise ratio S/N measured as a function of the detector thickness. The shaping time is $100 \mathrm{~ns}$.

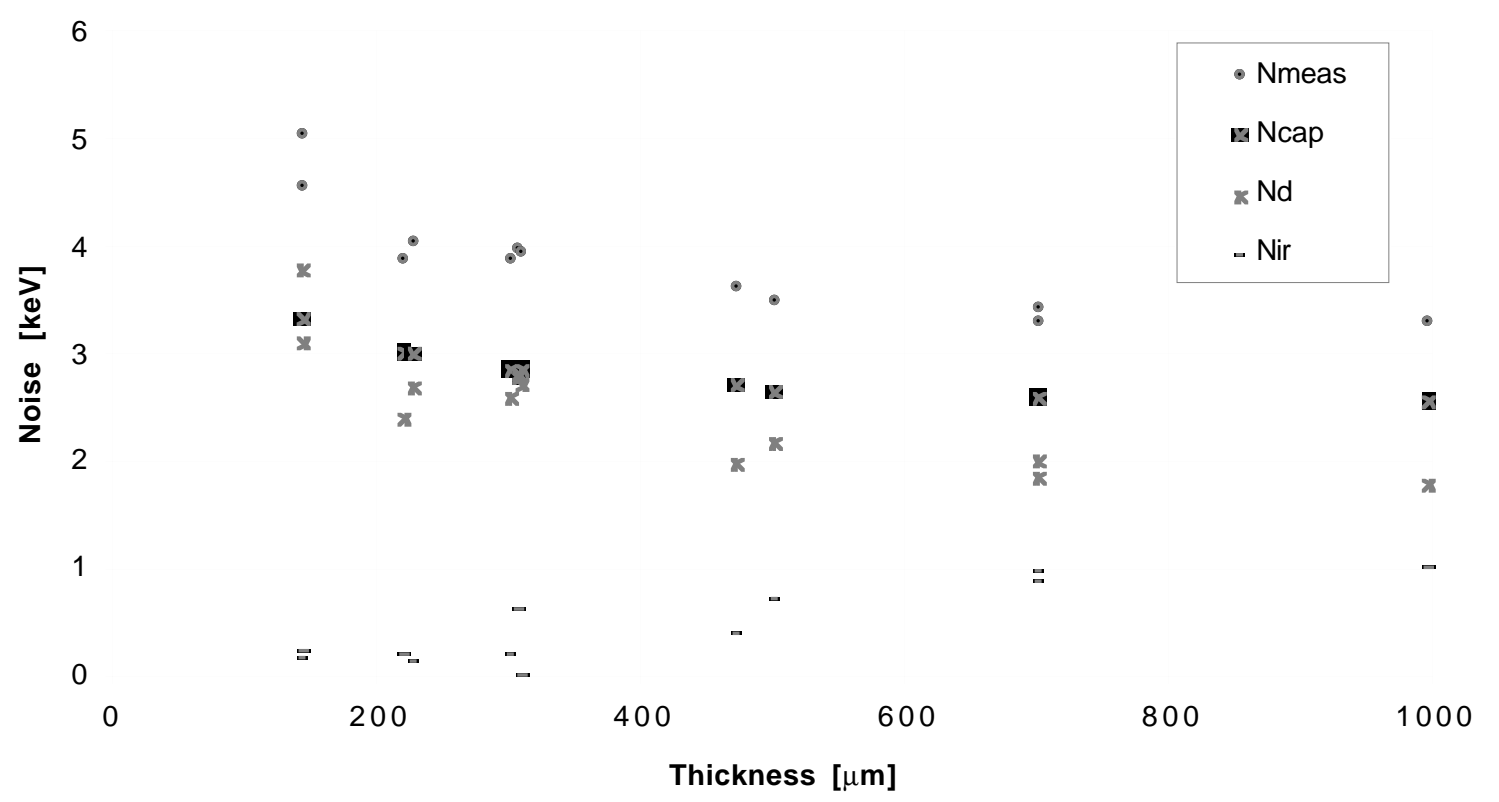

Fig. 2 The noise $\mathrm{N}_{\text {meas }}(\bullet)$ measured as a function the detector thickness; the capacitance noise $\mathrm{N}_{\text {cap }}(\boldsymbol{\square})$ is shown together with the reverse current noise, $\mathrm{N}_{\mathrm{Ir}},(-)$ and $\mathrm{N}_{\mathrm{d}}=\left(\mathrm{N}_{\text {meas }}-\mathrm{N}_{\text {cap }}^{2}-\mathrm{N}_{\text {Ir }}^{2}\right)^{1 / 2}(*)$. The shaping time is $100 \mathrm{~ns}$.

$\mathrm{N}_{\text {ir }}$ depends on the reverse current $\mathrm{I}_{\mathrm{r}}$ and on the shaping time $\theta$. It can be expressed as:

$$
\mathrm{N}_{\mathrm{ir}}=\left[\left(\mathrm{I}_{\mathrm{r}} \theta\right) / \mathrm{q}\right]^{1 / 2} \text {. }
$$

In the absence of irradiation, $\mathrm{I}_{\mathrm{r}}$ results from defects present in silicon introducing minority carriers. The energy levels associated with them act as traps. Thermal excitations may cause de- 
trapping that yields a reverse current which then depends on the temperature $(T)$ and the number of traps. The reverse current behaves as:

$$
\mathrm{I}_{\mathrm{r}} \propto \mathrm{N}_{\mathrm{t}} \mathrm{T}^{2} \exp \left(-\mathrm{E}_{\mathrm{g}} / 2 \mathrm{kT}\right)
$$

where $\mathrm{N}_{\mathrm{t}}$ is the trap concentration in silicon and $\mathrm{E}_{\mathrm{g}} \sim 1.2 \mathrm{eV}$ is the silicon energy band gap. The reverse current contribution to the total noise of non-irradiated detectors is small as shown in Fig. 2. The number of traps increases with the detector thickness and $\mathrm{N}_{\mathrm{ir}}$ is expected to increase with the detector thickness as observed in Fig. 2, although being negligible compared with $\mathrm{N}_{\text {cap }}$ which finally determines the noise level of non-irradiated detectors. The behaviour of $\mathrm{S} / \mathrm{N}$ and $\mathrm{N}$ with the detector thickness gives support for choosing detectors $\sim 300 \mu \mathrm{m}$ thick for the LHC [1].

For non-irradiated detectors, the relation between the applied bias voltage, $\mathrm{V}$, and the depletion thickness, $X_{d}$, is given by Eq. (2) if $V \leq V_{f d}$, where $V_{f d}$ is the full depletion voltage and

$$
X_{d}=w
$$

if $\mathrm{V} \geq \mathrm{V}_{\mathrm{fd}}$, where $\mathrm{w}$ is the full depletion depth.

Therefore, a signal proportional to the detector depletion width has a square-root dependence on the bias voltage below the full depletion voltage and is constant as a function of $\mathrm{V}$ above it. This voltage dependence is shown in Fig. 3a for a non-irradiated detector where the signal is plotted as a function of $(\mathrm{V})^{1 / 2}$. The dependence of the signal on the depletion voltage changes dramatically for irradiated detectors. The measurements were performed about five months after irradiation, the detectors being stored at room temperature (R.T.). The signal no longer has a square-root dependence on the bias voltage (Figs. 3b-3d). The changes in the dopant concentration (donors and/or acceptors) under irradiation [4] affect the value of the depletion voltage to be applied to achieve full depletion. Equation (6) remains unchanged but Eq. (2) must then be replaced by:

$$
\mathrm{X}_{\mathrm{d}}=\mathrm{f}(\mathrm{V})
$$

for $\mathrm{V} \leq \mathrm{V}_{\mathrm{fd}}$ as shown in Figs. 3b-3d where $\mathrm{f}(\mathrm{V})$ is a function of the applied voltage. The signal $\mathrm{S}$ can be fitted to a function $\mathrm{aV}^{\mathrm{n}} . \mathrm{n}$ is found to be 0.58 for a non-irradiated detector (Fig. $4 \mathrm{a}$ ) and reaches a value of up to 1.1 for a detector irradiated at a fluence of $1.2 \times 10^{14} \mathrm{p} / \mathrm{cm}^{2}$ (Fig. $4 \mathrm{~d}$ ).

The study of noise as a function of the shaping time $\theta$ reveals two components: the series noise and the parallel noise. The series noise behaves as $1 /(\theta)^{1 / 2}$ and is caused by thermal noise in the channel of the field-effect transistor which is the first amplifying stage in the preamplifier. It dominates the noise for short shaping times. The parallel noise arises from noise sources that are effectively in parallel with the detector at the preamplifier input i.e. in the present case the detector reverse current. Therefore, the parallel noise behaves as $(\theta)^{1 / 2}$ [Eq. (4)] and dominates the noise for long shaping times. The total noise as a function of the shaping time $\theta$ is:

$$
\mathrm{N}(\theta)=\mathrm{B} /(\theta)^{1 / 2}+\mathrm{D}(\theta)^{1 / 2} \text {. }
$$




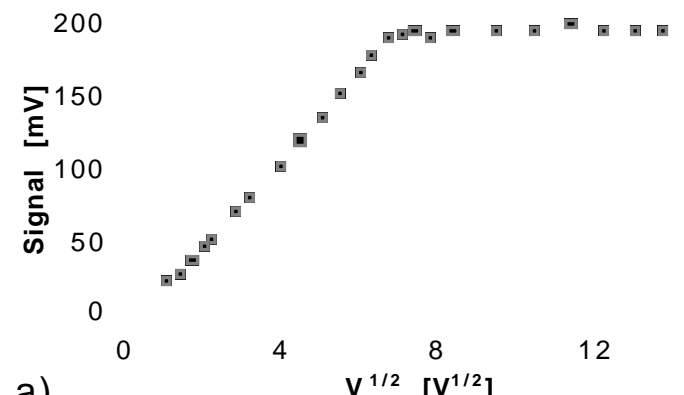

a)

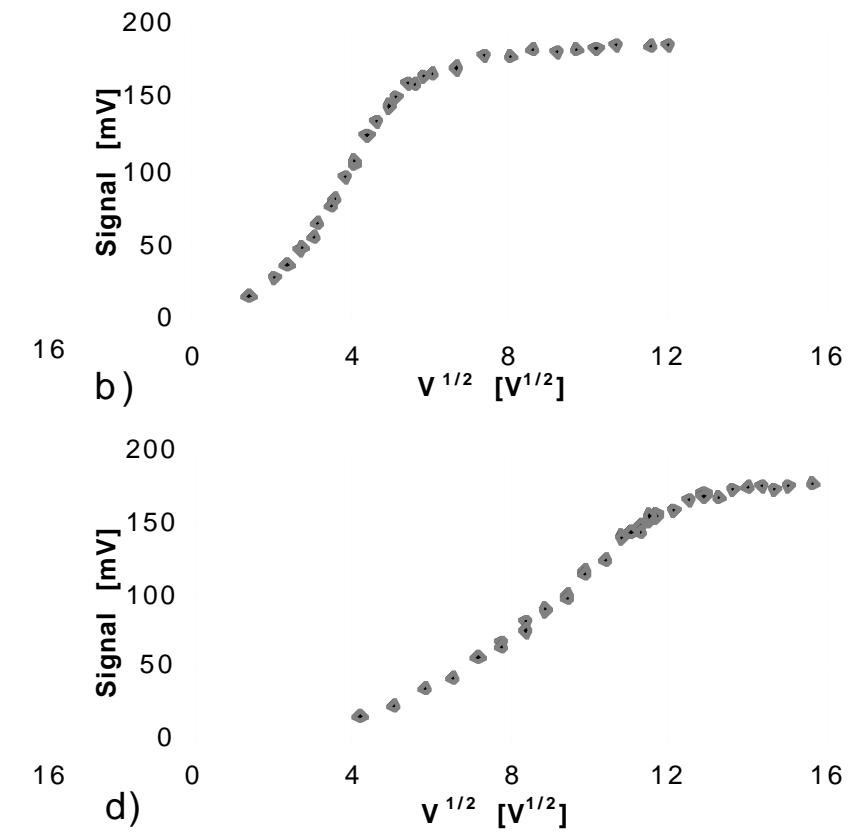

Fig. 3 The signal $(\mathrm{mV})$ as a function of the square root of the applied voltage for a non-irradiated detector (a) and detectors irradiated at various proton fluences: $2.8 \times 10^{13} \mathrm{p} / \mathrm{cm}^{2}$ (b), $6.2 \times 10^{13} \mathrm{p} / \mathrm{cm}^{2}(\mathrm{c}), 1.2 \times 10^{14} \mathrm{p} / \mathrm{cm}^{2}$ (d). The measurements were performed about five months after irradiation.

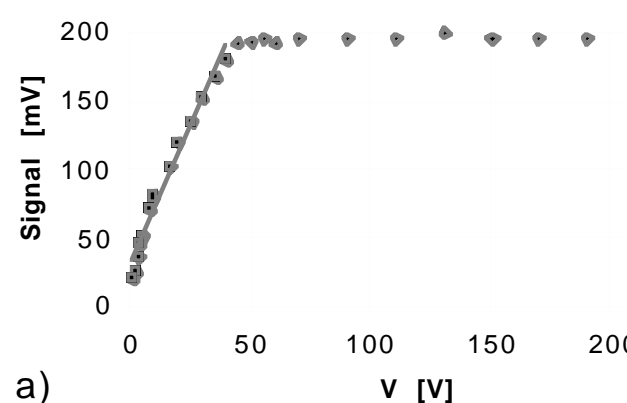

a)

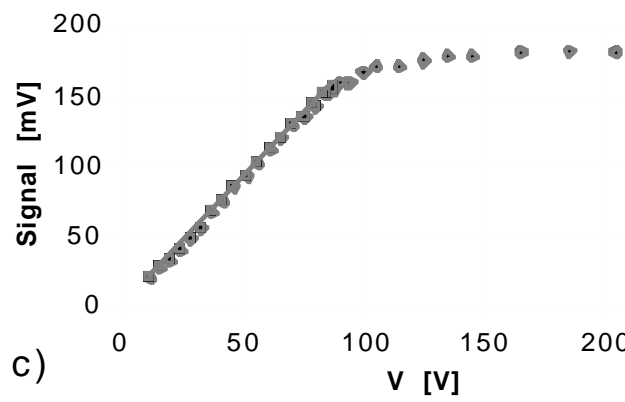

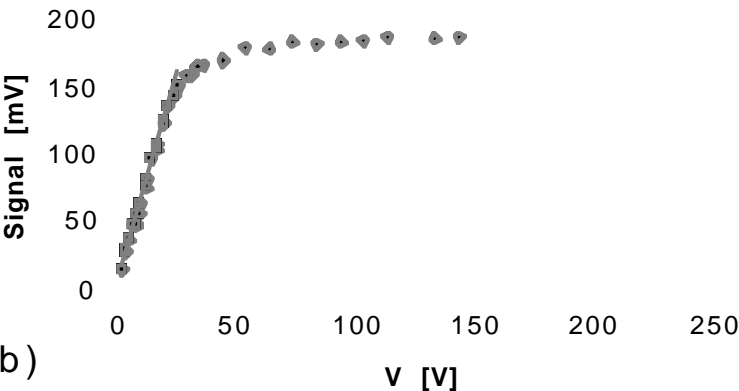

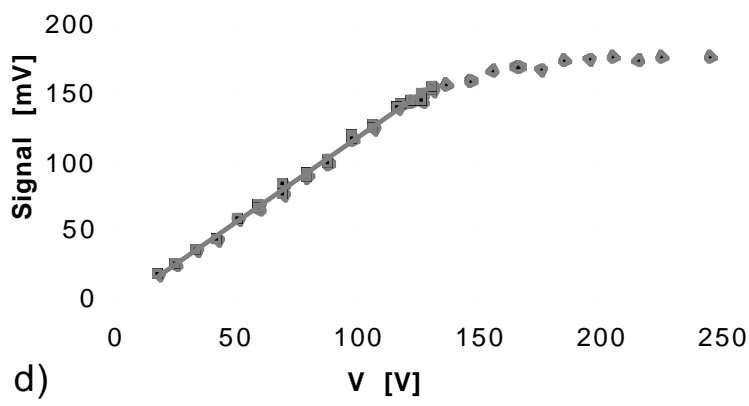

Fig. 4 The signal $(\mathrm{mV})$ as a function of the applied voltage for a non-irradiated detector (a) and detectors irradiated at various proton fluences: $2.8 \times 10^{13} \mathrm{p} / \mathrm{cm}^{2}(\mathrm{~b}), 6.2 \times 10^{13} \mathrm{p} / \mathrm{cm}^{2}(\mathrm{c}), 1.2 \times 10^{14} \mathrm{p} / \mathrm{cm}^{2}$ (d). The lines represent a fit to a function $\mathrm{aV}^{\mathrm{n}}$ with $\mathrm{n}=0.58$ (a), 0.99 (b), 1.05 (c), and 1.10 (d).

The series noise, originating from the preamplifier, can be estimated by identifying it with the noise measured for a non-irradiated detector $\left(\mathrm{N}_{\text {non-ir }}\right)$ for which $\mathrm{I}_{\mathrm{r}} \sim 0$. The parallel noise can be calculated from Eq. (4). Therefore, the total noise of an irradiated detector at a given shaping time can be obtained from:

$$
\mathrm{N}(\theta)=\left(\left(\mathrm{N}_{\text {non-ir }}\right)^{2}+\left(\mathrm{I}_{\mathrm{r}} \theta / \mathrm{q}\right)\right)^{1 / 2}
$$


Figure 5 shows the noise in number of electrons measured as a function of the shaping time for various fluences and temperatures. In Fig. 5, the noise for irradiated detectors as calculated from Eq. (9) is compared with the measured noise. The agreement with the measurements is excellent. It is observed that the series noise dominates the noise for $\theta \leq 50 \mathrm{~ns}$, while the parallel noise dominates for $\theta \geq 70 \mathrm{~ns}$. The noise of the irradiated detectors reaches a minimum in the region $30 \mathrm{~ns} \leq \theta \leq 50 \mathrm{~ns}$. The series noise which represents also the total noise of the non-irradiated detector $\left(\mathrm{I}_{\mathrm{r}} \sim 0\right)$ tends to a constant value for $\theta \geq 80 \mathrm{~ns}$. This constant value represents the minimum level of noise due to the readout electronics $\sim 1100-1200$ electrons. This level of the series noise and the size of the reverse current are the limiting factors for non-irradiated and irradiated detectors, respectively. The first could be reduced if improved electronics were to be used while the second can be reduced by operating the irradiated detectors at low temperature.

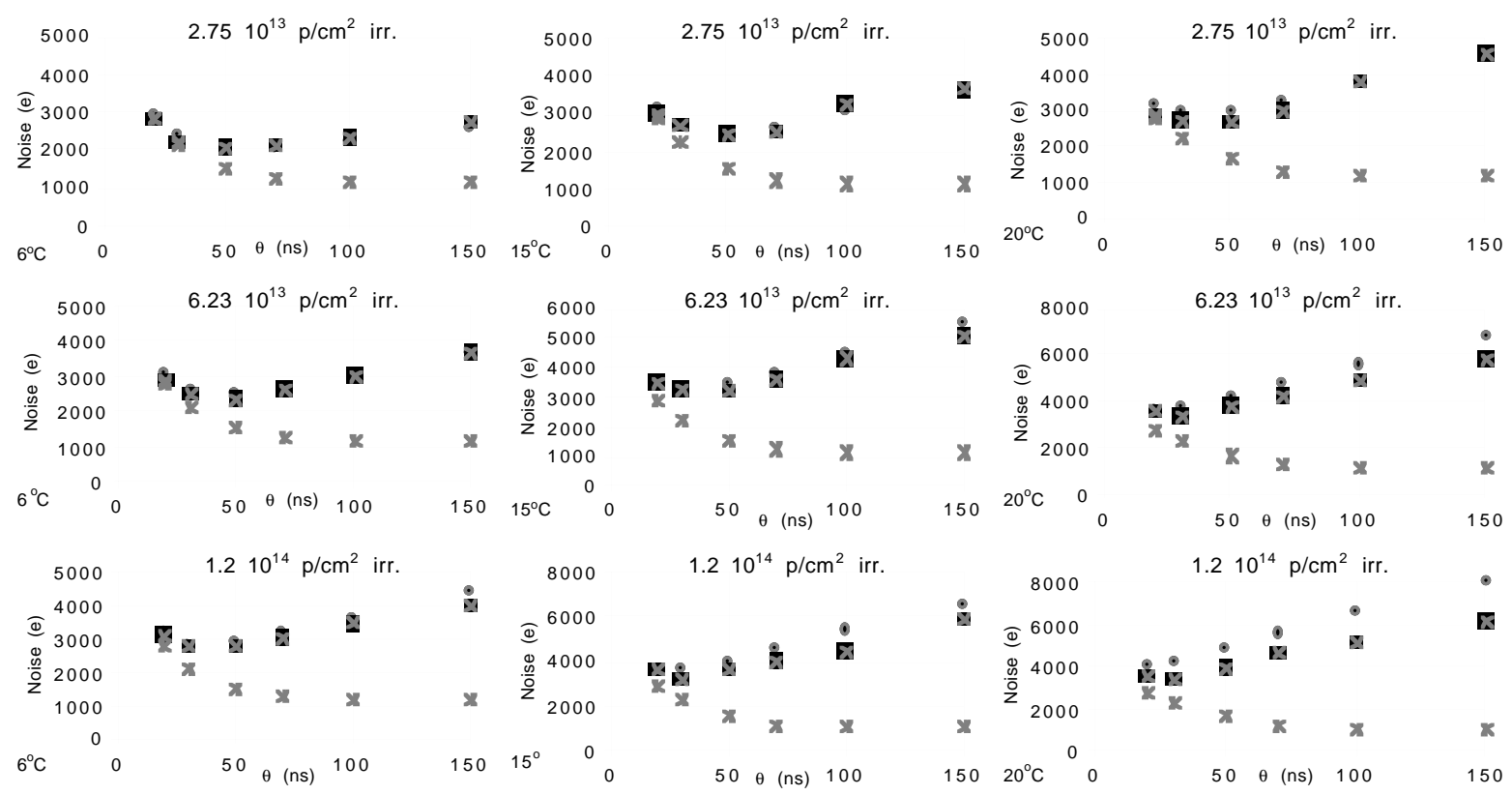

Fig. 5 The noise (in number of electrons) measured as a function of the shaping time $\theta$ for various fluences and temperatures. In each figure, the noise for irradiated detectors $(\bullet)$ as calculated from Eq. (9) (see text) is compared with the noise measured for irradiated detectors $(\mathbf{\square})$. The noise for the non-irradiated detector $\left(\mathrm{N}_{\text {non-ir }}\right)$ is also shown $(*)$.

The noise structure is reflected by $\mathrm{S} / \mathrm{N}$ which can be decomposed as a function of the shaping time:

$$
\mathrm{S} / \mathrm{N}=\mathrm{B}^{\prime}(\theta)^{1 / 2}+\mathrm{D}^{\prime} /(\theta)^{1 / 2}
$$

where $\mathrm{D}^{\prime} \sim\left(\mathrm{I}_{\mathrm{r}}\right)^{-1 / 2}$. S/N measured as a function of the shaping time for non-irradiated and irradiated detectors at various fluences is shown in Fig. 6 for $\mathrm{T}=6,15,20^{\circ} \mathrm{C}$. It is observed that $\mathrm{S} / \mathrm{N}$ for the irradiated detectors is maximum in the region $20 \mathrm{~ns} \leq \theta \leq 50 \mathrm{~ns}$ which corresponds to the region where the noise is minimal. For the non-irradiated detector, $\mathrm{S} / \mathrm{N} \sim 20,15$, and 10 for $\theta \geq 100 \mathrm{~ns}, \theta=50 \mathrm{~ns}$ and $\theta=30 \mathrm{~ns}$, respectively. $\mathrm{S} / \mathrm{N}$ for non-irradiated detectors is practically independent of temperature $\left(\mathrm{I}_{\mathrm{r}} \sim 0\right)$. For the irradiated detectors, $\mathrm{S} / \mathrm{N}$ decreases with an increasing fluence and strongly depends on temperature $\left(\mathrm{I}_{\mathrm{r}}\right.$ is large). For a fluence of $1.2 \times$ $10^{14} \mathrm{p} / \mathrm{cm}^{2}$ and $\mathrm{T}=6{ }^{\circ} \mathrm{C}, \mathrm{S} / \mathrm{N} \sim 5,7$, and 7 for $\theta \geq 100 \mathrm{~ns}, \theta=50 \mathrm{~ns}$ and $\theta=30 \mathrm{~ns}$, respectively. The $\mathrm{B}^{\prime}(\theta)^{1 / 2}$ component dominates for $\theta \leq 50$ ns while the $\mathrm{D}^{\prime} /(\theta)^{1 / 2}$ component dominates for $\theta \geq 100 \mathrm{~ns}$. 


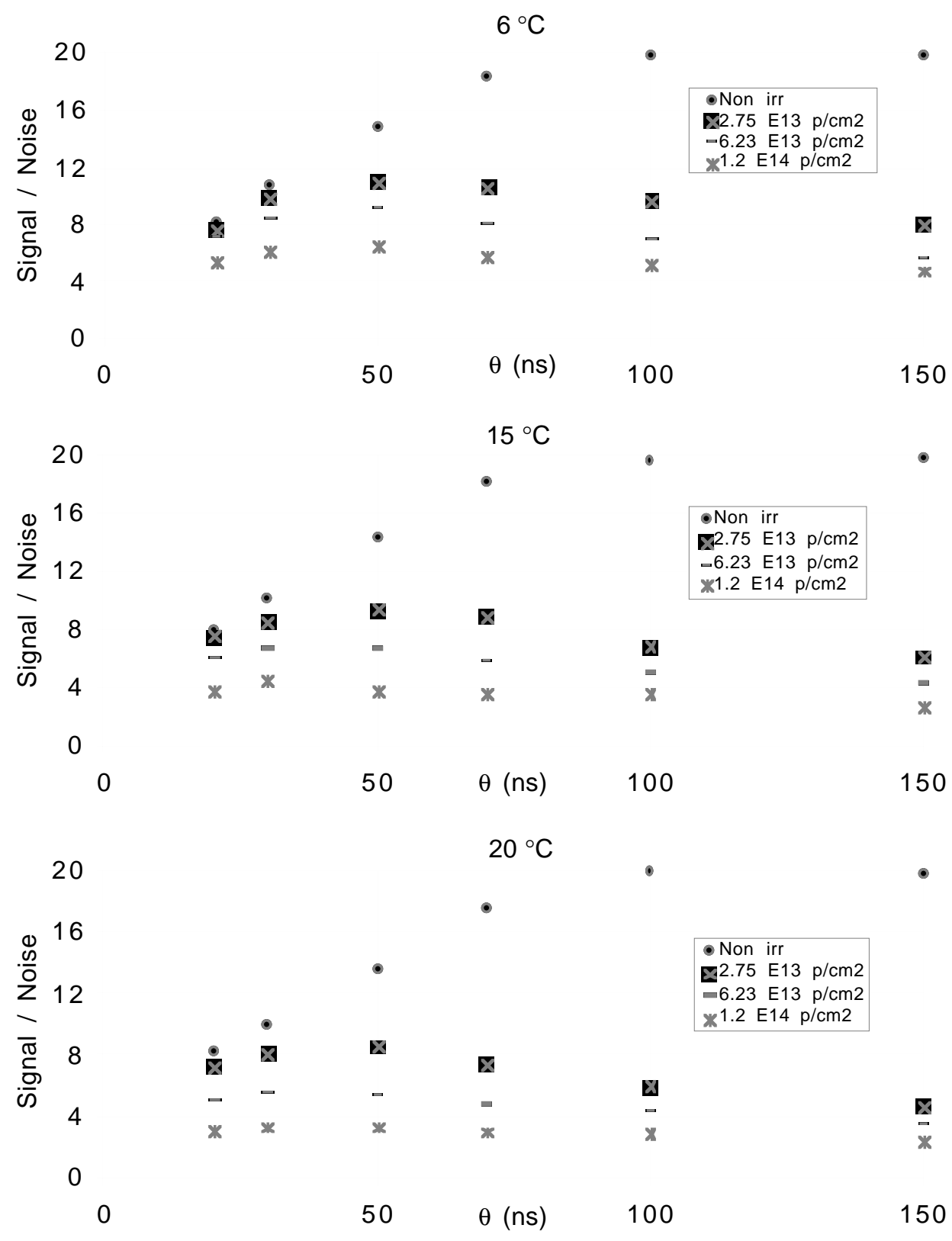

Fig. $6 \mathrm{~S} / \mathrm{N}$ measured as a function of the shaping time for a non-irradiated $(\bullet)$ and detectors irradiated at various fluences: $\left.\phi=2.8 \times 10^{13} \mathrm{p} / \mathrm{cm}^{2}(\mathbf{\square}), 6.2 \times 10^{13} \mathrm{p} / \mathrm{cm}^{2}(-), 1.2 \times 10^{14} \mathrm{p} / \mathrm{cm}^{2}{ }^{*}\right)$, for $\mathrm{T}=6,15,20^{\circ} \mathrm{C}$.

The signal as a function of the applied voltage is shown at $\mathrm{T}=6^{\circ} \mathrm{C}$ for long $(\theta=100 \mathrm{~ns})$ and short $(\theta=50,30 \mathrm{~ns})$ shaping times in Fig. 7 for the non-irradiated detector and in Fig. 8 for a detector irradiated at $\Phi=1.2 \times 10^{14} \mathrm{p} / \mathrm{cm}^{2}$. S/N can be calculated from these figures and found to be $\sim 4.0$ for $\theta=30 \mathrm{~ns}, \mathrm{~V} / \mathrm{V}_{\mathrm{fd}}=0.5, \Phi=1.2 \times 10^{14} \mathrm{p} / \mathrm{cm}^{2}$ and $\mathrm{T}=6{ }^{\circ} \mathrm{C}$ (Table 1). 


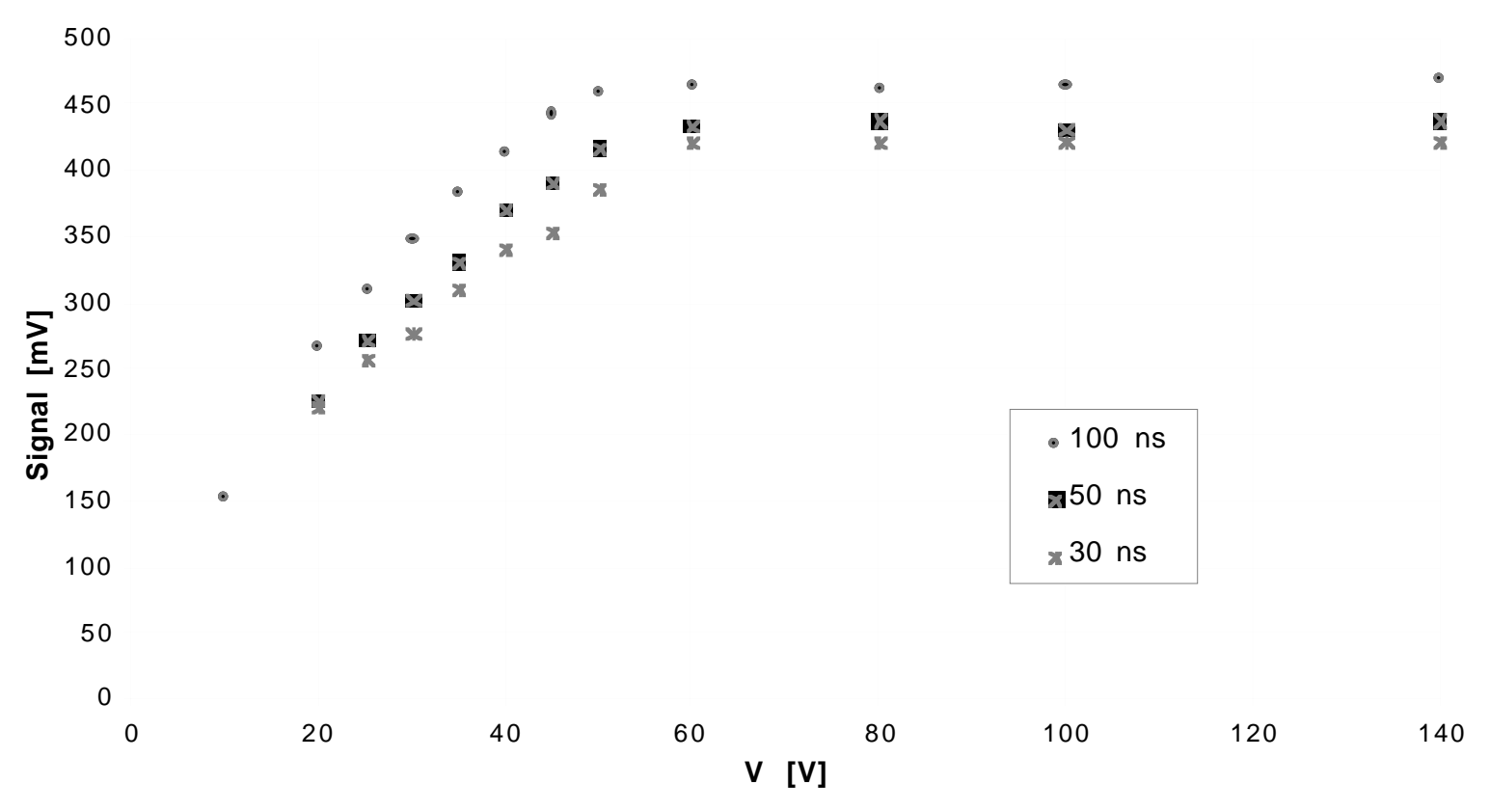

Fig. 7 The signal $\mathrm{S}(\mathrm{mV})$ of a non-irradiated detector measured as a function of the applied voltage for $\theta=100 \mathrm{~ns}$ $(\bullet) 50 \mathrm{~ns}(\boldsymbol{\square})$, and $30 \mathrm{~ns}(*)$. T $=6^{\circ} \mathrm{C}$.

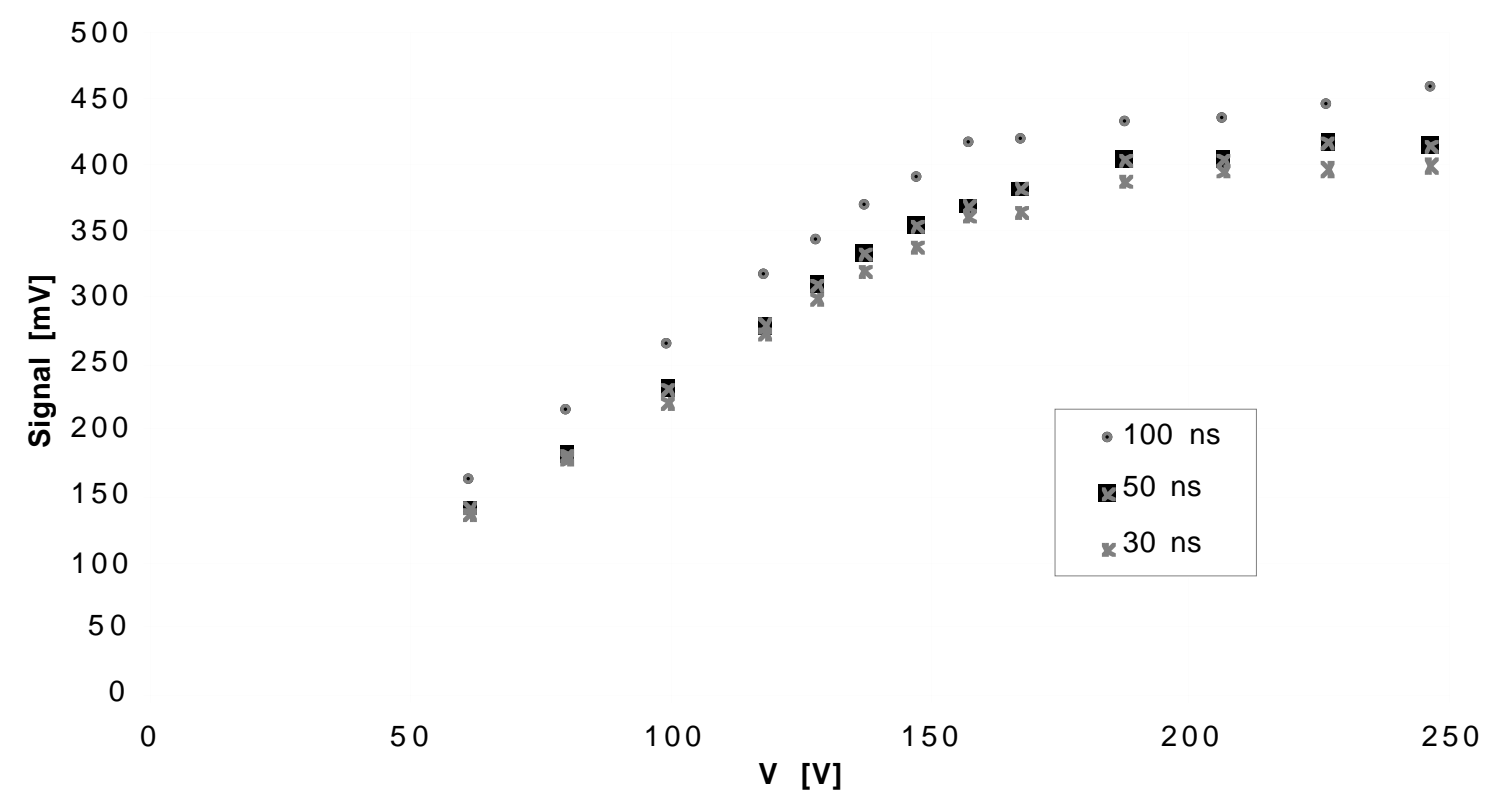

Fig. 8 The signal S (mV) of a detector irradiated at a proton fluence of $1.2 \times 10^{14} \mathrm{p} / \mathrm{cm}^{2}$ measured as a function of the applied voltage for $\theta=100 \mathrm{~ns}(\bullet), 50 \mathrm{~ns}(\boldsymbol{\square})$, and $30 \mathrm{~ns}(*) . \mathrm{T}=6^{\circ} \mathrm{C}$. 
Table 1

$\mathrm{S} / \mathrm{N}$ as a function of $\mathrm{V} / \mathrm{V}_{\mathrm{fd}}$ for various shaping times $(\theta=100,50,30 \mathrm{~ns})$ and fluences $(\phi)$ at $\mathrm{T}=6{ }^{\circ} \mathrm{C}$

\begin{tabular}{|c|c|c|c|c|}
\hline$\Phi$ & $\mathrm{V} / \mathrm{V}_{\mathrm{fd}}$ & $\mathrm{S} / \mathrm{N}(100 \mathrm{~ns})$ & $\mathrm{S} / \mathrm{N}(50 \mathrm{~ns})$ & $\mathrm{S} / \mathrm{N}(30 \mathrm{~ns})$ \\
\hline $1.2 \times 10^{14} \mathrm{p} / \mathrm{cm}^{2}$ & 0.50 & 3.8 & 3.9 & 3.6 \\
\hline $1.2 \times 10^{14} \mathrm{p} / \mathrm{cm}^{2}$ & 0.75 & 5.1 & 5.9 & 5.6 \\
\hline $1.2 \times 10^{14} \mathrm{p} / \mathrm{cm}^{2}$ & 1.00 & 6.0 & 7.4 & 7.2 \\
\hline 0.0 & 0.50 & 11.2 & 8.0 & 6.3 \\
\hline 0.0 & 0.75 & 16.3 & 13.3 & 9.1 \\
\hline 0.0 & 1.00 & 20.0 & 14.0 & 10.0 \\
\hline
\end{tabular}

\section{CONCLUSIONS}

The present study has been performed on large-area silicon detectors $\left(0.82\right.$ and $\left.1.0 \mathrm{~cm}^{2}\right)$ and mainly with a thickness of $300 \mu \mathrm{m}$. Non-irradiated and irradiated detectors (up to a fluence of $1.2 \times 10^{14} \mathrm{p} / \mathrm{cm}^{2}$ ) were used. The irradiation increases the size of the reverse current from nanoamperes for non-irradiated detectors to tens of microamperes for irradiated detectors (up to $\sim 100 \mu \mathrm{A}$ for $\Phi=1.2 \times 10^{14} \mathrm{p} / \mathrm{cm}^{2}$ at room temperature). This increase of reverse current introduces a strong temperature dependence of the noise. Then, working at low temperatures $\left(\leq 6{ }^{\circ} \mathrm{C}\right)$ has the consequence of limiting the reverse current $\left(\mathrm{I}_{\mathrm{r}}=70-100 \mu \mathrm{A}\right.$ at $\mathrm{T}=20{ }^{\circ} \mathrm{C}$ decreases to $\mathrm{I}_{\mathrm{r}}=45 \mu \mathrm{A}$ at $\mathrm{T}=15{ }^{\circ} \mathrm{C}$ and further to $\mathrm{I}_{\mathrm{r}}=20 \mu \mathrm{A}$ at $\mathrm{T}=6{ }^{\circ} \mathrm{C}$, for the detector irradiated at $\left.\phi=1.2 \times 10^{14} \mathrm{p} / \mathrm{cm}^{2}\right)$. It also limits the parallel noise $\left(\mathrm{N}_{\mathrm{ir}}=\left(\mathrm{I}_{\mathrm{r}} \theta / \mathrm{q}\right)^{1 / 2}\right)$ which dominates the total noise of irradiated detectors for $\theta \geq 70 \mathrm{~ns}$ and consequently increases $\mathrm{S} / \mathrm{N}$.

The noise and signal-to-noise ratio dependence on the shaping time have been studied over the range $20 \mathrm{~ns} \leq \theta \leq 150 \mathrm{~ns}$. The noise has two components: (a) the series noise which dominates the noise of non-irradiated detectors and the noise of irradiated detectors for $\theta \leq$ $50 \mathrm{~ns}$; (b) the parallel noise which depends on the reverse current and dominates the total noise of irradiated detectors for $\theta \geq 70 \mathrm{~ns}$. The size of the series noise and of the reverse current are the limiting factors for non-irradiated and irradiated detectors, respectively. The first could be reduced if improved electronics were to be used while the second can be reduced by operating the irradiated detectors at low temperature $\left(\mathrm{T} \leq 6^{\circ} \mathrm{C}\right)$.

The behaviour of the signal-to-noise ratio with the shaping time reflects the noise structure. For non-irradiated detectors, $\mathrm{S} / \mathrm{N} \sim 6.0$ and 10.0 at $\theta=30 \mathrm{~ns}$ for $\mathrm{V} / \mathrm{V}_{\mathrm{fd}}=0.5$ and 1.0, respectively. For detectors irradiated at a fluence of $1.2 \times 10^{14} \mathrm{p} / \mathrm{cm}^{2}$, i.e. 10 years of operation at the $\mathrm{LHC}, \mathrm{S} / \mathrm{N} \sim 4.0$ and 7.0 at $\theta=30 \mathrm{~ns}$ for $\mathrm{V} / \mathrm{V}_{\mathrm{fd}}=0.5$ and 1.0 , respectively. Therefore, $\mathrm{S} / \mathrm{N}$ is quite measurable even after 10 years of operation at the LHC and with a depletion voltage which is the half of the full depletion voltage at a shaping time of $30 \mathrm{~ns}$.

Finally, the signal-to-noise ratio measured for detectors irradiated at fluences corresponding to 10 years of operation at the LHC is not so different from the one measured for non-irradiated detectors when working at low temperature $\left(\mathrm{T} \leq 6^{\circ} \mathrm{C}\right)$ and with a shaping time of $30 \mathrm{~ns}$ corresponding to the LHC inter-bunch crossing time. This is the consequence of the 
fact that the noise of non-irradiated and irradiated detectors is dominated by the series noise for $\theta \sim 30 \mathrm{~ns}$. Therefore, a strong increase of the signal-to-noise ratio is expected at a shaping time of $\sim 30 \mathrm{~ns}$ if the series noise is reduced by using improved electronics which is presently under development [5].

\section{References}

[1] ATLAS: technical proposal for a general-purpose experiment at the Large Hadron Collider at CERN, CERN LHCC 94-43; LHCC P2.

[2] CMS: technical proposal for the Compact Muon Solenoid, CERN LHCC 94-38; LHCC P1.

[3] S.M. Sze, Physics of semiconductor devices, 2nd ed. (Wiley, New York, 1981).

[4] S.J. Bates et al., Recent results of radiation damage studies in silicon, Nucl. Instrum. Methods Phys. Res. A344 (1994) 228.

[5] A. Rudge, Comparison of charge collection in semiconductor detectors and timing resolution, using a sub-nanosecond transimpedance amplifier, Proceedings of the 6th Pisa Meeting on Advanced Detectors, La Biodola, Isola d'Elba, Italy, 22-28 May 1994, Nucl. Instrum. Methods Phys. Res. A360 (1995) 169. 